\title{
The variant undecapeptide sequence of the Arcanobacterium pyogenes haemolysin, pyolysin, is required for full cytolytic activity
}

\author{
Stephen J. Billington, J. Glenn Songer and B. Helen Jost
}

Department of Veterinary Science and Microbiology, The University of Arizona, 1117 East Lowell Street, Tucson, AZ 85721, USA
Author for correspondence: Stephen J. Billington. Tel: +1 520621 2745. Fax:+1 5206216366. e-mail: sbilling@u.arizona.edu

The cholesterol-dependent cytolysins (CDCs) are characterized by an undecapeptide sequence (ECTGLAWEWWR) that is located near the C terminus and within domain 4 of these proteins. Pyolysin (PLO), the CDC of Arcanobacterium pyogenes, has a variant undecapeptide sequence (EATGLAWDPWW). Site-directed mutants were constructed in undecapeptide residues in a recombinant PLO molecule containing a hexahistidine tag (HisPLO). Mutations in each of the three undecapeptide tryptophan residues resulted in low haemolytic activity, confirming the importance of these residues in the protein. Deletion of a proline residue $\left(\mathbf{P}_{499}\right)$, inserted in PLO, or substitution of this residue with either phenylalanine or glycine resulted in mutant proteins with undetectable or low haemolytic activities, indicating that $\mathbf{P}_{499}$ is essential for His-PLO haemolytic activity. Substitution of the PLO undecapeptide sequence with a consensus undecapeptide resulted in a His-PLO protein with only $0.1 \%$ activity, confirming that the variant PLO undecapeptide is required for the full cytolytic activity of this toxin. The presence of the conserved undecapeptide cysteine residue either alone (His-PLO.C ${ }_{492}$ ) or in a consensus sequence resulted in His-PLO molecules which were activated in the presence of reducing compounds, confirming the importance of this residue in the thiol-activated nature of many CDC toxins. The ability of His-PLO mutant proteins to bind cholesterol mimicked haemolytic activity, with the exception of His-PLO.C $\mathrm{C}_{422}$, which, despite having reduced haemolytic activity, showed an increased ability to bind cholesterol compared to His-PLO. Despite reductions in haemolytic activity and cholesterol-binding, all mutant proteins were still able to bind to erythrocyte membranes, suggesting that other regions of PLO may recognize host-cell membranes, through receptors other than cholesterol.

Keywords: cholesterol-dependent cytolysin, virulence factor, amino acid substitution

\section{INTRODUCTION}

Arcanobacterium pyogenes is commensal on the mucous membranes of the upper respiratory and urogenital tracts of cattle, sheep, swine and other domestic animals (Carter \& Chengappa, 1991). This organism is also commensal on the ruminal wall of cattle, from where it can participate in liver abscessation, secondary to ruminal acidosis (Narayanan at al., 1998). The recent isolation of $A$. pyogenes from the porcine gastric mucosa

Abbreviations: $\mathrm{BME}, \beta$-mercaptoethanol; $\mathrm{CDC}$, cholesterol-dependent cytolysin; ILY, intermedilysin; LLO, listeriolysin O; PFO, perfringolysin O; PLO, pyolysin; PLY, pneumolysin; SRBC, sheep red blood cell.
(Jost et al., 2002) suggests that it may also be a common inhabitant of the gastrointestinal tract of domestic animals. A. pyogenes is an important opportunistic pathogen of livestock and wild ungulates, causing diseases ranging from liver abscessation and mastitis in cattle (Jonsson et al., 1991; Lechtenberg et al., 1988; Nagaraja et al., 1996) to intracranial abscessation in white-tailed deer (Baumann et al., 2001).

A. pyogenes secretes a haemolytic exotoxin, pyolysin (PLO) (Billington et al., 1997; Ding \& Lämmler, 1996), which is an important virulence factor, as a knockout mutation in the plo gene resulted in reduced virulence of the mutant in a mouse model (Jost et al., 1999). Recombinant PLO is also an effective vaccine in the 
(a)

\begin{tabular}{|c|c|c|c|}
\hline SLO & 529 & $E C T G L A W E$ & W W \\
\hline PLY & 427 & $E C T G L A W E$ & w w \\
\hline SLY & 455 & $E C T G L A W E$ & W w \\
\hline LLO & 483 & $E C T G L A W E$ & W W \\
\hline LSO & 484 & $E C T G L F W E$ & W W \\
\hline ILO & 458 & $E C T G L A W E$ & W W \\
\hline PFO & 458 & $E C T G L A W E$ & W W F \\
\hline ALY & 460 & $E C T G L A W E$ & W w \\
\hline CLY & 465 & $E C T G L A W E$ & W W \\
\hline PLO & 491 & T G L A W & W w \\
\hline ILY & 485 & & \\
\hline
\end{tabular}

(b)

\begin{tabular}{|c|c|}
\hline $\begin{array}{l}\text { His-PLO } \\
\text { His-PLO.C } 492\end{array}$ & $\begin{array}{l}E A T G L A W D P W W \text { - } \\
E C T G L A W D P W W-\end{array}$ \\
\hline His-PLO.F.F & EATGLAFDPWW - \\
\hline His-PLO.F ${ }_{500}$ & EATGLAWD \\
\hline His-PLO.F 501 & E A T G L AWD \\
\hline His-PLO. $\Delta P_{499} \Omega R_{502}$ & $E A T G L A W D$ \\
\hline His-PLO. $\Omega R_{502}$ & E A T G L AWD \\
\hline His-PLO. $\Delta P_{499}$ & E A T G L A WD \\
\hline His-PLO.F 499 & $E A T G L A W D$ \\
\hline His-PLO.G & w w \\
\hline His-PLO & \\
\hline His-PLO & $\overline{E A} T G$ \\
\hline
\end{tabular}

Fig. 1. Alignment of $C D C$ undecapeptide sequences and undecapeptide substitutions in mutant His-PLO molecules. (a) Amino acid sequence alignment of the undecapeptide sequence of CDCs. Amino acids which match the consensus undecapeptide sequence are highlighted. Amino acid numbers are given for the first amino acid in the sequence for each protein. The amino acid sequences of streptolysin molecules from Streptococcus canis, Streptococcus dysgalactiae subsp. equisimilis and Streptococcus pyogenes are identical over the region shown. SLO, streptolysin O; SLY: suilysin; LSO, seeligerilysin O; ILO, ivanolysin O; ALY, alveolysin; CLY, cereolysin. (b) Amino acid sequence alignment showing amino acid substitutions in the undecapeptide of His-PLO constructed by site-directed mutagenesis. Substituted amino acids are highlighted.

prevention of experimental infections in mice (Jost et al., 1999), although its ability to protect domestic animals from natural infection is still to be tested. PLO is a novel member of a group of toxins historically known as the thiol-activated cytolysins. As all members of this family are not subject to thiol-activation, including PLO (Billington et al., 1997; Nagamune et al., 1996), a number of alternative designations have been proposed (Billington et al., 2000; Gilbert et al., 1999; Nagamune, 1997; Shatursky et al., 1999), with cholesterol-dependent cytolysins (CDCs) (Shatursky et al., 1999) being the most widely accepted. This group of toxins, which includes listeriolysin $\mathrm{O}$ (LLO), perfringolysin $\mathrm{O}$ (PFO), pneumolysin (PLY) and streptolysin O, is characterized by an undecapeptide sequence (ECTGLAWEWWR) located near the $\mathrm{C}$ terminus of the protein (Fig. 1a). Oxidation of the cysteine residue within this sequence is responsible for the thiol-activated nature of many CDCs. Both PLO and intermedilysin (ILY) of Streptococcus intermedius have variant undecapeptide sequences, in which the conserved cysteine has been substituted with an alanine residue, resulting in oxygenresistant toxins (Billington et al., 1997; Nagamune et al., 1996; Nagamune, 1997) (Fig. 1a). Many advances have been made in recent years as to the function of the four domains within the CDC tertiary structures and their roles in pore-formation (Tweten et al., 2001). However, while it has been evident for some time that the undecapeptide is critical for CDC activity (Boulnois et al., 1991; de los Toyos et al., 1996; Jacobs et al., 1999; Korchev et al., 1998; Michel et al., 1990; Pinkney et al., 1989; Saunders et al., 1989; Sekino-Suzuki et al., 1996), the precise function of this region of the molecule is unclear.

The crystal structure of the prototype CDC, Clostridium perfringens PFO, has been described as an elongated molecule composed of four $\beta$-sheet-rich domains, only one of which, the C-terminal domain 4 , is contiguous within the primary amino acid sequence (Rossjohn et al., 1997). A proposed structure for S. pneumoniae PLY based on a homology model with PFO indicates that PLY takes on a very similar structure (Rossjohn et al., 1998) and it appears likely, given the conservation of residues that map to the hydrophobic core of PFO, that all CDCs, including PLO, share a similar three-dimensional structure (Rossjohn et al., 1997). Studies of pore formation by PFO (Heuck et al., 2000; Shatursky et al., 1999; Shepard et al., 1998, 2000) indicate that two transmembrane amphipathic $\beta$-hairpins (TMHs) from domain 3 span the membrane bilayer and serve as the membrane-aqueous interface of the PFO pore. In the crystal structure of the PFO monomer, the tryptophanrich undecapeptide region forms a hydrophobic loop at one end of domain 4 (Rossjohn et al., 1997), initially proposed to extend out, as a result of cholesterol binding, to form a dagger which facilitates membrane insertion (Rossjohn et al., 1997). However, it has recently been demonstrated that domain 4 does not penetrate deeply into the lipid bilayer, but is responsible for the initial interaction of CDCs with the host membrane (Heuck et al., 2000). In fact, the conformational changes required to form the domain 3 TMHs do not take place until domain 4 has partially inserted into the lipid bilayer (Heuck et al., 2000). Thus, domain 4 may act to sample the membrane for suitability or to tether monomers to the membrane in a pre-pore complex, prior to the insertion of the TMHs (Heuck et al., 2000; Hotze et al., 2002). The undecapeptide sequence has been implicated in the initial binding of CDCs to host-cell membranes, as mAbs directed against the undecapeptide sequence block binding of PLY to erythrocyte membranes (Jacobs et al., 1999), and sitedirected mutations in the undecapeptide sequences, particularly the conserved tryptophan residues, of many CDCs significantly affect the ability of these toxins to 
form pores (Boulnois et al., 1991; Michel et al., 1990; Sekino-Suzuki et al., 1996).

Both PLO and the human-specific cytolysin ILY have variant undecapeptide sequences (Billington et al., 1997; Nagamune, 1997) (Fig. 1a). It is not known if this sequence divergence represents an attempt to regulate cytolytic activity or whether, in the case of PLO which is the most divergent member of the CDC family, the sequence divergence compensates for structural differences in other regions of the protein between PLO and the other CDCs. In this study, we examine the activity of various site-specific mutants in the undecapeptide of PLO and determine that the sequence variation in the PLO undecapeptide is required for full cytolytic activity.

\section{METHODS}

Bacterial strains and growth conditions. Escherichia coli strains $\mathrm{DH} 5 \alpha$ and $\mathrm{DH} 5 \alpha \mathrm{F}^{\prime}$ lacl $^{\mathrm{q}}$ (Gibco-BRL) were grown either on Luria-Bertani (LB) agar or in LB broth (Difco) at $37^{\circ} \mathrm{C}$. Ampicillin $\left(100 \mu \mathrm{g} \mathrm{ml}^{-1}\right)$ was added to the medium when appropriate.

DNA techniques. Procedures for E. coli transformation and plasmid extraction, DNA restriction, ligation, agarose gel electrophoresis and Southern blotting were performed as described by Ausubel et al. (1994). Plasmids pJGS59, encoding a 543 aa protein (His-PLO) composed of 507 aa of the mature PLO with an N-terminal extension of 36 aa encoded by pTrcHis B, including a hexahistidine sequence, and pJGS89, a site-directed mutant of pJGS59 that encodes a mutant version of His-PLO with an alanine to cysteine change at position 492 (His-PLO.C ${ }_{492}$ ), have been described previously (Billington et al., 1997). pJGS59 and pJGS89 were used as templates for the construction of site-directed mutants, using the mutagenic primers indicated in Table 1 and the Transformer Site Directed Mutagenesis Kit (Clontech), according to the manufacturer's instructions. Two selection primers were used. Primer $5^{\prime}-$ GACTTGGTTGAGGCCTCACCAGTCAC-3' was designed to change the $S c a$ I site of pTrcHis B to StuI and was used in conjunction with forward primers listed in Table 1. A second selection primer, 5'-GAGTGCACCATGGGCGGTGTGAAAT-3', was designed to change the $\mathrm{NdeI}$ site of p TrcHis B to $\mathrm{NcoI}$ and was used in conjunction with the reverse primers listed in Table 1. Introduction of appropriate mutations into either pJGS59 or pJGS89 was confirmed by automated DNA sequencing on a 373A DNA sequencer (Applied Biosystems) at the University of Arizona's DNA sequencing facility.

Production and purification of His-PLO and mutant proteins. Cultures $(500 \mathrm{ml})$ of E. coli $\mathrm{DH} 5 \alpha$ or $\mathrm{DH} 5 \alpha \mathrm{F}^{\prime} l a c I^{\mathrm{q}}$ carrying pJGS59, or the appropriate site-directed mutant plasmid, were grown to an $\mathrm{OD}_{600}$ value of 0.6 prior to induction with $2.5 \mathrm{mM}$ IPTG for 3 h. Cells were harvested by centrifugation at $5000 \mathrm{~g}$ and the cell pellet was resuspended in binding buffer (20 mM Tris $/ \mathrm{HCl}, 100 \mathrm{mM} \mathrm{NaCl}, \mathrm{pH} \mathrm{8} 0)$. The cells were disrupted by two passages through a French Pressure Cell (Aminco) at $138 \mathrm{MPa}$ and the insoluble material was removed by centrifugation at $12000 \mathrm{~g}$. His-PLO or mutant proteins were purified from the soluble fraction using TALON Metal Affinity Resin (Clontech) according to the manufacturer's instructions, and eluted from the column in binding buffer with $50 \mathrm{mM}$ imidazole. Protein concentration was determined using the Bradford Protein Assay Reagent (Bio-Rad).

Haemolytic assays. Microtitration haemolytic assays were performed essentially as described previously (Billington et al., 1997), except that dilutions of toxins were performed in $1 \mathrm{mg}$ BSA ml ${ }^{-1}$ to aid in stabilization of the toxin. One haemolytic unit (HU) was defined as the amount of haemolysin (in ng) required to release $50 \%$ of the haemoglobin from $200 \mu \mathrm{l}$ of $0.25 \%$ sheep red blood cells (SRBCs) in $1 \mathrm{~h}$ at $37^{\circ} \mathrm{C}$, and specific haemolytic activity was expressed as HU ( $\mu \mathrm{g}$ protein $)^{-1}$. The specific haemolytic activity of all His-PLO mutant proteins was expressed as a percentage of His-PLO haemolytic activity, which was designated as $100 \%$. The effects of reducing compounds on haemolytic activity was assayed by incubating $10 \mu \mathrm{g}$ toxin preparations $\mathrm{ml}^{-1}$ with final concentrations of either $2.5 \mathrm{mM}$ DTT or $2 \% \quad(\mathrm{v} / \mathrm{v}) \beta$-mercaptoethanol (BME) for $10 \mathrm{~min}$ at room temperature, prior to performance of the haemolytic assay.

Cholesterol-binding assays. The relative cholesterol-binding affinity of His-PLO and mutant proteins was assayed by a modification of an ELISA technique developed by Shimada et

Table 1. Construction of site-specific undecapeptide mutants in His-PLO

\begin{tabular}{|c|c|c|}
\hline Toxin & Plasmid & Mutagenic primer* \\
\hline His-PLO & pJGS59 & $\begin{array}{ccccccccccc}E & \text { A } & \text { T } & \text { G } & \text { L } & \text { A } & \text { W } & \text { D } & \text { P } & \text { W } & \text { W } \dagger \\
5^{\prime}-\text { GAGGCAACTGGCCTAGCGTGGGATCCGTGGTGG-3' }\end{array}$ \\
\hline His-PLO.C ${ }_{492}$ & pJGS89 & 5'-GAAGCAGGCGAGTGCACTGGCCTAGC-3' \\
\hline His-PLO.F $F_{497}$ & pJGS135 & 5'-GCCTAGCGTTCGATCCGTGG-3' \\
\hline His-PLO.F $F_{500}$ & pJGS187 & 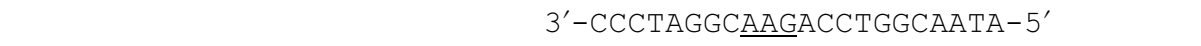 \\
\hline His-PLO.F $F_{501}$ & pJGS199 & 3'-CCCTAGGCACCAAGTGGCAATA-5' \\
\hline His-PLO. $\Delta \mathrm{P}_{499} \Omega \mathrm{R}_{502}$ & pJGS138 & 3'-GCACCCTA $\underline{---A C C A C C G C T T G G C A A T A G-5^{\prime}}$ \\
\hline His-PLO. $\Omega \mathrm{R}_{502}$ & pJGS215 & 3'-CTAGGCACCACCECTTGGCAATAGTTAT-5' \\
\hline His-PLO. $\Delta \mathrm{P}_{499}^{.02}$ & pJGS214 & $3^{\prime}-$ GATCGCACCCTA---ACCACCTGGC-5' \\
\hline His-PLO.F $\mathrm{F}_{499}$ & pJGS257 & 3'-CGCACCCTAAAGACCACCTGGC-5' \\
\hline His-PLO.G ${ }_{499}$ & pJGS258 & 3'-CGCACCCTACCGACCACCTGGC-5' \\
\hline His- $\mathrm{PLO}_{\mathrm{CON}}$ & pJGS210 & 5'-GAAGCAGGCGAGTGCACTGGCCTAGC-3' \\
\hline His-PLO ${ }_{\mathrm{CON}+\mathrm{A}}$ & pJGS209 & $\begin{array}{l}\text { 3'-GCACCCTC---ACCACCGCTTGGCAATAG-5' } \\
\text { 3'-GCACCCTC---ACCACCGCTTGGCAATAG-5' }\end{array}$ \\
\hline
\end{tabular}

*Underlined sequences represent mutated codons.

† The His-PLO undecapeptide sequence and its corresponding DNA sequence are shown. 
al. (1999). Microtitre plate wells were coated with $1 \mu \mathrm{mol}$ of cholesterol, overnight at $37^{\circ} \mathrm{C}$. Following two washes in $0 \cdot 1 \mathrm{M}$ PBS ( $\mathrm{pH} 7 \cdot 2$ ) containing $0 \cdot 3 \%(\mathrm{v} / \mathrm{v})$ Tween 20 (PBS-T), the wells were blocked for $1 \mathrm{~h}$ with $3 \%(\mathrm{w} / \mathrm{v})$ BSA in PBS at $4{ }^{\circ} \mathrm{C}$. The wells were washed twice with PBS-T and incubated for $1 \mathrm{~h}$ at $37^{\circ} \mathrm{C}$ with dilutions of toxin in $1 \mathrm{mg} \mathrm{BSA} \mathrm{ml}{ }^{-1}(1 \mu \mathrm{g}$ to $1 \mathrm{ng}$ toxin or, where necessary, $10 \mu \mathrm{g}$ to $10 \mathrm{ng}$ toxin). Following nine washes with PBS-T, bound toxin was reacted with a 1/500 dilution of polyclonal goat anti-PLO serum (Billington et al., 1997) at $37^{\circ} \mathrm{C}$ for $1 \mathrm{~h}$. Primary antibody was detected using a horseradish-peroxidase-conjugated rabbitanti-goat $\operatorname{IgG}(\mathrm{H}+\mathrm{L})$ peroxidase $(\mathrm{KPL})$ and $o$-phenylenediamine as substrate (Sigma).

The cholesterol-binding activity was calculated as the amount of haemolysin (in $\mathrm{ng}$ ) required to give an ELISA OD ${ }_{420}$ value $50 \%$ that given by $1 \mu \mathrm{g}$ His-PLO. The binding activity of all His-PLO mutant proteins was expressed as a percentage of His-PLO binding activity, which was designated as $100 \%$. An ELISA was also performed on known amounts of each mutant toxin to confirm that His-PLO and each mutant reacted equally with the anti-PLO serum.

Membrane-binding assays. These were performed essentially as described by de los Toyos et al. (1996). His-PLO and mutant toxins were diluted in $1 \mathrm{mg} \mathrm{BSA} \mathrm{ml}{ }^{-1}$ to a concentration of $2.5 \mu \mathrm{g} \mathrm{ml}^{-1}$. Toxin $(0.5 \mathrm{ml})$ was added to an equal volume of $10 \%$ SRBC and incubated on ice for $20 \mathrm{~min}$. SRBCs were pelleted by centrifugation at $14000 \mathrm{~g}$ for $5 \mathrm{~min}$ at $4{ }^{\circ} \mathrm{C}$. Cell pellets were lysed by boiling in sample buffer and were then subjected to SDS-PAGE and Western blot analysis with the anti-PLO serum to detect bound toxin.

\section{RESULTS}

The tryptophan residues of PLO are important in cytolytic activity

Mutations in any of the hydrophobic tryptophan residues of the undecapeptide have significant effects on the pore-forming ability of LLO, PFO and PLY (Boulnois et al., 1991; Korchev et al., 1998; Michel et al., 1990; Sekino-Suzuki et al., 1996). To determine if this was also true for PLO, each of the three tryptophan residues was changed individually to phenylalanine to create His-PLO.F $F_{497}$, His-PLO.F $F_{500}$ and His-PLO.F $F_{501}$ (Fig. 1b). Haemolytic assays were performed on purified protein from each of these mutants (Table 2). Like other CDCs, each mutant had significantly reduced haemolytic activity compared to wild-type His-PLO. The most severe reduction was observed with His-PLO.F $\mathrm{F}_{497}$, with only $0 \cdot 2 \%$ of His-PLO activity. The haemolytic activity of this mutant protein is consistent with observations from other CDCs, which suggest that the first tryptophan residue of the undecapeptide is essential for cytolytic activity (Boulnois $\mathrm{et}$ al., 1991; Sekino-Suzuki et al., 1996). The haemolytic activities of His-PLO.F $F_{500}$ and His-PLO.F $F_{501}$ at 2.3 and $5.0 \%$ of wild-type His-PLO activity, respectively, also suggest that these residues are important for PLO activity.

\section{The proline residue in the PLO undecapeptide is essential for activity}

The major structural and charge differences between the PLO undecapeptide and the consensus undecapeptide for the CDC family appear to be the insertion of a proline $\left(\mathrm{P}_{499}\right)$, which increases the spacing between the three tryptophan residues, and the lack of the terminal arginine (Fig. 1a). A mutant His-PLO protein was created in which the proline was deleted and the arginine inserted (His-PLO. $\Delta \mathrm{P}_{499} \Omega \mathrm{R}_{502}$ ) (Fig. 1b). This mutant protein showed only $2.5 \%$ haemolytic activity compared to wild-type His-PLO (Table 2). To determine which of the two mutations was having the greatest effect on activity, the arginine insertion (His-PLO $\Omega \mathrm{R}_{502}$ ) and proline deletion (His-PLO. $\Delta \mathrm{P}_{499}$ ) mutations were introduced independently into His-PLO (Fig. 1b). The HisPLO. $\Omega R_{502}$ mutant protein, while reduced in activity compared to His-PLO, still had significant haemolytic activity at $27.5 \%$ of wild-type (Table 2 ). The HisPLO. $\Delta \mathrm{P}_{499}$ protein, however, had no detectable haemolytic activity, even at toxin concentrations greater than $2 \mathrm{mg} \mathrm{ml}^{-1}$. Thus, the relative haemolytic activity of the protein was less than $0.003 \%$ of wild-type His-PLO. These data suggest that $\mathrm{P}_{499}$ plays an essential role in the cytolytic activity of PLO. To confirm the importance of $\mathrm{P}_{499}$, site-specific mutants were created where the proline was substituted with either phenylalanine (HisPLO. $F_{499}$ ) or glycine (His-PLO.G ${ }_{499}$ ) (Fig. 1b). His-

Table 2. Mean haemolytic and cholesterol-binding activities of His-PLO mutant proteins

\begin{tabular}{|lcc|}
\hline Protein & Haemolytic activity* & Cholesterol-binding activity* $^{*}$ \\
\hline His-PLO & $100 \cdot 00 \pm 12 \cdot 36$ & $100 \cdot 00 \pm 8 \cdot 82$ \\
His-PLO.F & $0 \cdot 22 \pm 0 \cdot 03$ & $1 \cdot 60 \pm 0 \cdot 26$ \\
His-PLO.F & & $7 \cdot 15 \pm 0 \cdot 93$ \\
His-PLO.F & $2 \cdot 34 \pm 0 \cdot 42$ & $7 \cdot 50 \pm 3 \cdot 06$ \\
His-PLO. $\Delta \mathrm{P}_{499} \Omega \mathrm{R}_{502}$ & $5 \cdot 00 \pm 0 \cdot 69$ & $4 \cdot 17 \pm 0 \cdot 70$ \\
His-PLO. $\Omega \mathrm{R}_{502}$ & $2 \cdot 46 \pm 0 \cdot 12$ & $26 \cdot 23 \pm 0 \cdot 31$ \\
His-PLO. $\Delta \mathrm{P}_{499}$ & $27 \cdot 50 \pm 1 \cdot 71$ & $<0 \cdot 06$ \\
His-PLO.F & $<0 \cdot 003$ & $5 \cdot 93 \pm 1 \cdot 12$ \\
His-PLO.G & $1 \cdot 30 \pm 0 \cdot 09$ & $0 \cdot 30 \pm 0 \cdot 06$ \\
His-PLO & $0 \cdot 36 \pm 0 \cdot 04$ & $1 \cdot 02 \pm 0 \cdot 25$ \\
\hline
\end{tabular}

* Haemolytic and cholesterol-binding activities are expressed as a percentage of His-PLO activity from an average of three experiments $\pm 1 \mathrm{sD}$ from the mean. 
Table 3. Effects of reducing compounds on the haemolytic and cholesterol-binding activities of cysteine-containing His-PLO mutant proteins

\begin{tabular}{|c|c|c|c|c|c|}
\hline \multirow[t]{2}{*}{ Protein* } & \multicolumn{3}{|c|}{ Haemolytic activity $\dagger$} & \multicolumn{2}{|c|}{ Cholesterol-binding activity $\dagger$} \\
\hline & Untreated & $+\mathrm{BME} \ddagger$ & + DTT & Untreated & $+\mathrm{BME} \neq$ \\
\hline His-PLO & $100 \cdot 00 \pm 12 \cdot 36$ & $100 \cdot 78 \pm 13 \cdot 70$ & $104 \cdot 58 \pm 14 \cdot 94$ & $100 \cdot 00 \pm 8 \cdot 82$ & $98 \cdot 03 \pm 5 \cdot 67$ \\
\hline His-PLO.C ${ }_{492}$ & $4 \cdot 88 \pm 0 \cdot 98$ & $44 \cdot 18 \pm 2 \cdot 94$ & $34 \cdot 67 \pm 2 \cdot 40$ & $\mathrm{ND}$ & $166 \cdot 20 \pm 43 \cdot 56$ \\
\hline His-PLO ${ }_{\mathrm{CON}}$ & $0 \cdot 14 \pm 0 \cdot 0$ & $1 \cdot 15 \pm 0 \cdot 05$ & $1 \cdot 03 \pm 0.06$ & ND & $2 \cdot 90 \pm 0.70$ \\
\hline
\end{tabular}

ND, Not determined.

*All protein preparations were stored at $4{ }^{\circ} \mathrm{C}$ for 2 weeks, prior to performing haemolytic assays.

$\dagger$ The haemolytic and cholesterol-binding activities are expressed as a percentage of His-PLO activity from an average of three experiments \pm 1 sD from the mean.

$\ddagger$ Proteins were pre-incubated with $2 \%(\mathrm{v} / \mathrm{v})$ BME for $10 \mathrm{~min}$ at room temperature, prior to performing haemolytic or cholesterol-binding assays.

\$Proteins were pre-incubated with $2.5 \mathrm{mM}$ DTT for $10 \mathrm{~min}$ at room temperature, prior to performing haemolytic assays.

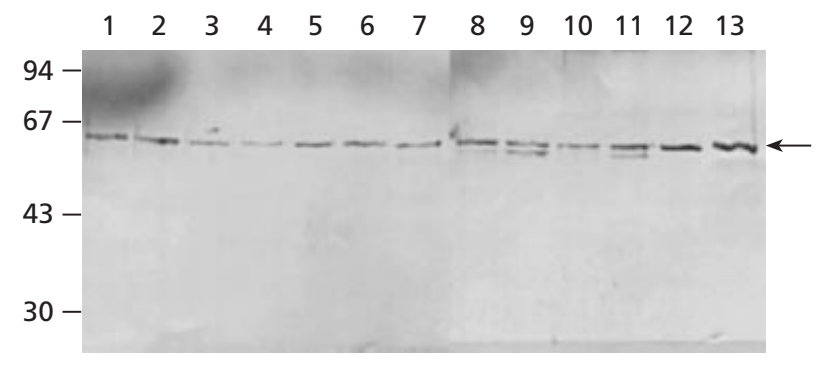

Fig. 2. Membrane binding of His-PLO mutant proteins. His-PLO and mutant toxins (both $1.25 \mu \mathrm{g}$ ) were incubated with $10 \%$ SRBCs on ice for $20 \mathrm{~min}$. SRBCs were pelleted, lysed by boiling in sample buffer and subjected to SDS-PAGE and Western blot analysis with anti-PLO serum to detect bound toxin. Lanes: 1, His-PLO; 2, His-PLO.F 497 ; 3, His-PLO.F $\mathrm{F}_{500} ; 4$, His-PLO.F ${ }_{501}$; 5, His-

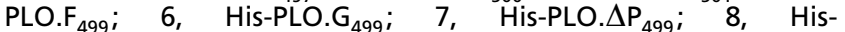
PLO. $\Delta \mathrm{P}_{499} \Omega \mathrm{R}_{502} ; 9$, His-PLO. $\Omega \mathrm{R}_{502} ; 10$, His-PLO $\mathrm{CON}+\mathrm{A} ; 11$, His$\mathrm{PLO}_{\text {CON }}+\mathrm{BME}$; 12 , His-PLO.C ${ }_{492}+\mathrm{BME} ; 13$, His-PLO+BME. The positions of molecular mass markers are shown in KDa on the left of the image; the arrow on the right indicates the position of the wild-type and mutant His-PLO proteins.

PLO.F 499 and His-PLO.G H99 $_{49}$ were also impaired for haemolytic activity, with relative haemolytic activities of 1.3 and $0.4 \%$ of His-PLO haemolytic activity, respectively (Table 2 ).

\section{The variation in the PLO undecapeptide is required for full cytolytic activity}

Since there are other changes in the PLO undecapeptide that may compensate for the proline and arginine changes, a consensus undecapeptide was introduced into His-PLO. This protein was constructed in two forms, one containing a purely consensus CDC undecapeptide, His-PLO ${ }_{\mathrm{CON}}$, which includes the conserved cysteine residue and another, $\mathrm{His}-\mathrm{PLO}_{\mathrm{CON}+\mathrm{A}}$, containing all the consensus residues but retaining the alanine of PLO at position 492 (Fig. 1b). Purified His- PLO $_{\text {CON }}$

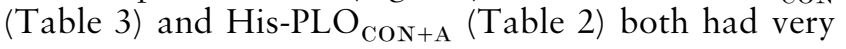

low haemolytic activities of $0 \cdot 1 \%$. This result confirmed that the substituted residues in the PLO undecapeptide were required for full cytolytic activity of this protein. Since His-PLO ${ }_{\mathrm{CON}}$ contained the conserved cysteine residue responsible for the thiol-activation of many CDCs, haemolytic assays were performed with purified protein pre-treated with either DTT or BME. In the presence of these reducing agents, the haemolytic activity of His- $\mathrm{PLO}_{\mathrm{CON}}$ was increased, approximately 10 -fold over no treatment, to $1 \cdot 0-1 \cdot 1 \%$ of His-PLO activity (Table 3 ). While this activity is still very low, it represents a significant increase in activity over unreduced protein and indicates that $\mathrm{His}-\mathrm{PLO}_{\mathrm{CON}}$ is subject to oxygen inactivation.

\section{Thiol-activation of His-PLO. $\mathrm{C}_{492}$}

The two members of the CDC family that are not subject to thiol-activation, PLO and ILY, each have divergent undecapeptide sequences, which include substitution of the conserved cysteine residue with an alanine residue (Billington et al., 1997; Nagamune, 1997). We have previously presented evidence that a His-PLO molecule in which the alanine residue has been changed to the conserved cysteine residue (HisPLO. $\mathrm{C}_{492}$ ) is not subject to oxygen inactivation (Billington et al., 1997) (Fig. 1b). However, our results that the activity of His-PLO ${ }_{\mathrm{CON}}$ can be activated by reducing agents led us to re-examine the activity of HisPLO.C ${ }_{492}$. Our initial experiments were performed on freshly prepared His-PLO and His-PLO.C C $_{492}$ (Billington et al., 1997). However, haemolytic assays performed on purified His-PLO.C ${ }_{492}$ following its storage for a 2-week period at $4{ }^{\circ} \mathrm{C}$ indicated that the haemolytic activity of this protein had been reduced to only $4.9 \%$ of wild-type His-PLO. This activity could be restored by pretreatment with either BME or DTT to 44.2 and $34.7 \%$ of His-PLO activity, respectively (Table 3), which approximates the activity originally observed with freshly purified His-PLO. $C_{492}$ (Billington et al., 1997). There- 
fore, contrary to our previous results (Billington et al., 1997), PLO can be engineered to be thiol-activated by the introduction of the conserved cysteine residue into the undecapeptide but, even when activated, this mutant is not as active as wild-type His-PLO.

\section{Cholesterol-binding activities of His-PLO mutant proteins}

Cholesterol is the major host-cell-surface receptor for CDCs and the undecapeptide region of these toxins has been implicated either in direct binding of cholesterol or in membrane insertion as a result of cholesterol binding. Each of the His-PLO mutant proteins was compared with His-PLO for its ability to bind to cholesterol in an ELISA-based assay. The ability of all mutant toxins, with the exception of His-PLO. $\mathrm{C}_{492}$, to bind cholesterol was in direct proportion to the haemolytic activity of the toxin (Tables 2 and 3). For example, His-PLO. $\Omega R_{502}$ had a haemolytic activity of $27.5 \%$ and a cholesterol-binding activity of $26.2 \%$ compared to His-PLO, while HisPLO. $F_{497}$ had 0.2 and $1.6 \%$ of His-PLO haemolytic and cholesterol-binding activities, respectively. Reduced His-PLO.C ${ }_{492}$, however, appeared to bind cholesterol as well as, if not better than, wild-type His-PLO (mean = $166 \cdot 2 \%$ His-PLO cholesterol-binding activity), despite having a haemolytic activity only $44 \cdot 2 \%$ that of HisPLO (Table 3).

\section{Membrane binding of His-PLO mutant proteins}

Cholesterol is the proposed major target cell receptor for CDCs, although it is not known if initial interactions occur through other host molecules. To test the ability of the mutant toxins to bind to host-cell membranes, SRBCs incubated with each toxin were analysed by Western blotting using anti-PLO serum (Fig. 2). Despite the variations in haemolytic activity (undetectable to $44.2 \%$ of His-PLO) and cholesterol binding (undetectable to $166 \cdot 2 \%$ of His-PLO), all mutant toxins bound to SRBC membranes, and no obvious defects in binding compared to His-PLO could be determined from this assay.

\section{DISCUSSION}

The undecapeptide of CDCs has for some time been implicated in the cytolytic activity of these toxins. This region is the largest stretch of identity among the toxins that originally formed the thiol-activated cytolysin family. However, with the discovery of the variant undecapeptide sequences of PLO (Billington et al., 1997) and ILY (Nagamune, 1997), the requirement for identity within this undecapeptide region to produce a functional cytolysin has been questioned (Billington et al., 2000). The haemolytic activity of PLO against SRBCs is approximately $10 \%$ that of PFO (S. J. Billington, unpublished observation), so it is possible that the variant undecapeptide of PLO is responsible for this reduced activity. However, PLO is the most divergent member of the CDC family (Billington et al., 1997), and it is also possible that the variations in the PLO undecapeptide sequence are required to compensate for structural differences imposed on the undecapeptide by other regions of the molecule. To assess the requirement for its variant undecapeptide, we have introduced various mutations into the undecapeptide of His-PLO. All mutations introduced had a deleterious effect on the cytolytic activity of His-PLO. In particular, the introduction of a consensus undecapeptide resulted in very low haemolytic activity, indicating that the variant undecapeptide of PLO is required for its full cytolytic activity.

Of the changes in the undecapeptide sequence of PLO compared to the CDC consensus, the proline insertion appears to have the greatest effect, since removal of this amino acid resulted in a protein that was non-haemolytic, even at very high toxin concentrations. The importance of $\mathrm{P}_{499}$ may be to provide the correct spacing between important residues, such as the three undecapeptide tryptophan residues. However, the substitution of $\mathrm{P}_{499}$ with either the similarly bulky amino acid phenylalanine or the flexible amino acid glycine did not result in functional proteins, suggesting that spacing alone does not explain the importance of $\mathrm{P}_{499}$. Proline residues are important in the structural integrity of many proteins, as they can force peptide bends. In fact, the general conservation of proline residues within CDC primary sequences suggests that they may be involved in the maintenance of higher-order structures (Billington et al., 1997). The undecapeptide proline residue may be required to align the PLO undecapeptide to compensate for structural differences imposed on the PLO undecapeptide by other regions of the PLO molecule, which vary considerably in primary amino acid sequence from the structural backbone of other CDCs. Interestingly, ILY, which is not nearly as divergent in its primary sequence, also contains a proline at the same position in the undecapeptide as PLO (Fig. 1a; Nagamune, 1997). However, in ILY the terminal arginine is retained and the third tryptophan residue is missing, which may have implications for its binding to host cells. Indeed, ILY appears to show more specificity in host-cell type than other members of the CDC family (Nagamune, 1997; Nagamune et al., 1996).

Despite the differences in the PLO undecapeptide, it shares the three hydrophobic tryptophan residues which have been demonstrated to play a pivotal role in the cytolytic activity of many CDCs (Boulnois et al., 1991; Korchev et al., 1998; Michel et al., 1990; Nakamura et al., 1998; Sekino-Suzuki et al., 1996). However, the spacing between the first and second tryptophan is altered by insertion of $\mathrm{P}_{499}$. Mutations in each of the tryptophan residues resulted in vastly reduced cytolytic activity in LLO, PFO and PLY (Boulnois et al., 1991; Korchev et al., 1998; Michel et al., 1990; Sekino-Suzuki et al., 1996). Consistent with results from other CDCs, substitution of any of the tryptophan residues of PLO with phenylalanine resulted in a substantial reduction in haemolytic activity compared to the wild-type, suggesting that the undecapeptide plays a similar role in PLO as it does in other members 
of the family. The role of the undecapeptide sequence in the initial binding, tethering or membrane sampling of CDCs has yet to be proven. However, the position of the first undecapeptide tryptophan residue at the very tip of the undecapeptide loop (Rossjohn et al., 1997) implies a prominent role in any initial interaction with the target cell membrane precipitated by domain 4 . The marked effect of mutations at this position in PLO (Table 2) and other CDCs (Boulnois et al., 1991; Sekino-Suzuki et al., 1996) supports the importance of this residue in CDC cytolytic activity.

Contrary to results with PFO (Sekino-Suzuki et al., 1996; Shimada et al., 1999), mutations in undecapeptide amino acids of His-PLO that resulted in decreased cytolytic activity, with the exception of the alanine to cysteine change in His-PLO.C C $_{492}$, also resulted in a concomitant reduction in cholesterol binding. There are several possible explanations for this observation. The undecapeptide of PLO may be intimately involved in cholesterol binding or mutations in undecapeptide residues may directly affect the cholesterol-binding site of PLO. Alternatively, mutations in the PLO undecapeptide may have a considerable effect on the structure of the PLO molecule, in particular domain 4, which likely contains the cholesterol-binding domain, such that it can no longer bind cholesterol. The variant nature of the PLO undecapeptide may be required not only to properly align the undecapeptide sequence but also to maintain the structure of domain 4 in general. Support for the latter comes from minor substitutions at the C terminus of CDCs (Owen et al., 1994; Shimada et al., 1999), which disrupt the ability of these toxins to bind to cholesterol and host-cell membranes. Aligned with this final suggestion is the possibility that mutations introduced in this study result in the misfolding of domain 4, or indeed the entire PLO protein structure, leading to the reduction in haemolytic and cholesterolbinding activities. This is not the case with the majority of mutations in this region of PFO (Sekino-Suzuki et al., 1996), where reduction in haemolytic activity was observed in the absence of major structural changes. Despite the reduced ability to bind to cholesterol, each of the His-PLO mutant toxins was still able to bind to erythrocyte membranes. These results suggest that CDCs may also bind through additional host-cell molecules (Dubail et al., 2001; Owen et al., 1994). This suggestion is supported by experiments with a truncated LLO molecule composed of domains 1, 2 and 3 but lacking the cholesterol-binding domain 4 , which indicated that this truncated molecule could still bind to host-cell membranes (Dubail et al., 2001).

The thiol-activated nature of the original members of the CDC family is the result of oxidation of the cysteine residue at position 2 of the undecapeptide motif (Michel et al., 1990; Pinkney et al., 1989; Saunders et al., 1989). Generally, mutagenesis of this cysteine residue to alanine resulted in a toxin with a similar cytolytic activity, but which was not inactivated by oxygen (Michel et al., 1990; Pinkney et al., 1989; Saunders et al., 1989). The identification of PLO and ILY confirmed speculation by
Pinkney et al. (1989) as to the presence of members of the CDC family that lack the cysteine residue and thus would be able to stably express their cytolytic activity. The significance of the thiol-activation of CDCs is unknown. It may be a fortuitous event in evolution, but the simplicity of the substitution to an oxygen-resistant form suggests some selection for the oxygen-sensitive form. It is possible that oxygen sensitivity may regulate toxin function following secretion by pathogenic bacteria in the host, although expression of an oxygenstable form of LLO had no effect on the pathogenesis of Listeria monocytogenes in mice (Michel et al., 1990). The His-PLO. $\mathrm{C}_{492}$ toxin, while reduced for haemolytic activity, appeared to bind cholesterol better than wildtype His-PLO. Perhaps in a molecule more amenable to its presence, the cysteine aids in the interaction of domain 4 with the primary host-cell receptor. Anecdotally, the His-PLO. $\mathrm{C}_{492}$ protein loses activity much more slowly than His-PLO ${ }_{\mathrm{CON}}$, although both lose approximately $90 \%$ of activity to oxygen inactivation. This observation may support the initial assertion that the variant sequence of the PLO undecapeptide affects the stability of PLO to oxygen, even in the presence of the cysteine substitution (Billington et al., 1997).

We conclude that the variant undecapeptide of PLO is required for full cytolytic activity of this toxin. It seems likely that the altered residues in the PLO undecapeptide may compensate for structural changes in other parts of PLO, with respect to other CDCs, as PLO has the most divergent amino acid sequence (Billington et al., 1997). It is also possible that the undecapeptide controls hostcell-membrane sampling. PLO is known to more-readily lyse erythrocytes from some animals species (e.g. rabbit or sheep) than other species (e.g. bovine) (Funk et al., 1996). This suggestion has implications for the variant undecapeptide of ILY and its limited host range (Nagamune, 1997; Nagamune et al., 1996).

\section{ACKNOWLEDGEMENTS}

This work was supported by NRICGP/USDA award 9935204-7818. The authors thank Hien T. Trinh for purification of the His-tagged proteins and Dawn M. Bueschel for excellent technical assistance.

\section{REFERENCES}

Ausubel, F. M., Brent, R., Kingston, R. E., Moore, D. D., Seidman, J. G., Smith, J. A. \& Struhl, K. (1994). Current Protocols in Molecular Biology. New York: Greene Publishing Associates and Wiley.

Baumann, C. D., Davidson, W. R., Roscoe, D. E. \& Beheler-Amass, K. (2001). Intracranial abscessation in white-tailed deer of North America. J Wildl Dis 37, 661-670.

Billington, S. J., Jost, B. H., Cuevas, W. A., Bright, K. R. \& Songer, J. G. (1997). The Arcanobacterium (Actinomyces) pyogenes hemolysin, pyolysin, is a novel member of the thiol-activated cytolysin family. J Bacteriol 179, 6100-6106.

Billington, S. J., Jost, B. H. \& Songer, J. G. (2000). Thiol-activated cytolysins: structure, function and role in pathogenesis. FEMS Microbiol Lett 182, 197-205.

Boulnois, G. J., Paton, J. C., Mitchell, T. J. \& Andrew, P. W. (1991). 
Structure and function of pneumolysin, the multifunctional, thiol-activated toxin of Streptococcus pneumoniae. Mol Microbiol 5, 2611-2616.

Carter, G. R. \& Chengappa, M. M. (1991). Essentials of Veterinary Bacteriology and Mycology, 4th edn. Philadelphia, PA: Lea \& Febiger.

de los Toyos, J. R., Mendez, F. J., Aparicio, J. F. \& 7 other authors (1996). Functional analysis of pneumolysin by use of monoclonal antibodies. Infect Immun 64, 480-484.

Ding, H. \& Lämmler, C. (1996). Purification and further characterization of a haemolysin of Actinomyces pyogenes. Zentralbl Veterinarmed (B) 43, 179-188.

Dubail, I., Autret, N., Beretti, J.-L., Kayal, S., Berche, P. \& Charbit, A. (2001). Functional assembly of two membrane-binding domains in listeriolysin $\mathrm{O}$, the cytolysin of Listeria monocytogenes. Microbiology 147, 2679-2688.

Funk, P. G., Staats, J. J., Howe, M., Nagaraja, T. G. \& Chengappa, M. M. (1996). Identification and partial characterization of an Actinomyces pyogenes hemolysin. Vet Microbiol 50, 129-142.

Gilbert, R. J. C., Jimenez, J. L., Chen, S., Tickle, I. J., Rossjohn, J., Parker, M., Andrew, P. W. \& Saibil, H. R. (1999). Two structural transitions in membrane pore formation by pneumolysin, the pore-forming toxin of Streptococcus pneumoniae. Cell 97, 647-655.

Heuck, A. P., Hotze, E. M., Tweten, R. K. \& Johnson, A. E. (2000) Mechanism of membrane insertion of a multimeric $\beta$-barrel protein: perfringolysin $\mathrm{O}$ creates a pore using ordered and coupled conformational changes. Mol Cell 6, 1233-1242.

Hotze, E. M., Heuck, A. P., Czajkowsky, D. M., Shao, Z., Johnson, A. E. \& Tweten, R. K. (2002). Monomer-monomer interactions drive the prepore to pore conversion of a $\beta$-barrel-forming cholesterol-dependent cytolysin. J Biol Chem 277, 11597-11605.

Jacobs, T., Cima-Cabal, M. D., Darji, A. \& 7 other authors (1999). The conserved undecapeptide shared by thiol-activated cytolysins is involved in membrane binding. FEBS Lett 459, 463-466.

Jonsson, P., Olsson, S.-O., Olofson, A.-S., Fälth, C., Holmberg, O. \& Funke, H. (1991). Bacteriological investigations of clinical mastitis in heifers in Sweden. J Dairy Res 58, 179-185.

Jost, B. H., Songer, J. G. \& Billington, S. J. (1999). An Arcanobacterium (Actinomyces) pyogenes mutant deficient in production of the pore-forming cytolysin pyolysin has reduced virulence. Infect Immun 67, 1723-1728.

Jost, B. H., Post, K. W., Songer, J. G. \& Billington, S. J. (2002). Isolation of Arcanobacterium pyogenes from the porcine gastric mucosa. Vet Res Commun 26, 419-425.

Korchev, Y. E., Bashford, C. L., Pederzolli, C., Pasternak, C. A. Morgan, P. J., Andrew, P. W. \& Mitchell, T. J. (1998). A conserved tryptophan in pneumolysin is a determinant of the characteristics of channels formed by pneumolysin in cells and planar lipid bilayers. Biochem J 329, 571-577.

Lechtenberg, K. F., Nagaraja, T. G., Leipold, H. W. \& Chengappa, M. M. (1988). Bacteriologic and histologic studies of hepatic abscesses in cattle. Am J Vet Res 49, 58-62.

Michel, E., Reich, K. A., Favier, R., Berche, P. \& Cossart, P. (1990) Attenuated mutants of the intracellular bacterium Listeria monocytogenes obtained by single amino acid substitution in listeriolysin O. Mol Microbiol 4, 2167-2178.

Nagamune, H. (1997). Streptococcal cytolysins. Seikagaku 69 , 343-348.

Nagamune, H., Ohnishi, C., Katsuura, A., Fushitani, K., Whiley, R. A., Tsuji, A. \& Matsuda, Y. (1996). Intermedilysin, a novel cytotoxin specific for human cells, secreted by Streptococcus intermedius UNS46 isolated from a human liver abscess. Infect Immun 64, 3093-3100.

Nagaraja, T. G., Laudert, S. B. \& Parrott, J. C. (1996). Liver abscesses in feedlot cattle. Part I. Causes, pathogenesis, pathology, and diagnosis. Comp Cont Edu Pract Vet 18, S230-S241, S256.

Nakamura, M., Sekino-Suzuki, N., Mitsui, K.-I. \& Ohno-Iwashita, Y. (1998). Contribution of tryptophan residues to the structural changes in perfringolysin $\mathrm{O}$ during interaction with liposomal membranes. J Biochem 123, 1145-1155.

Narayanan, S., Nagaraja, T. G., Wallace, N., Staats, J., Chengappa, M. M. \& Oberst, R. D. (1998). Biochemical and ribotypic comparison of Actinomyces pyogenes and A.pyogenes-like organisms from liver abscesses, ruminal wall, and ruminal contents of cattle. Am J Vet Res 59, 271-276.

Owen, R. H. G., Boulnois, G. J., Andrew, P. W. \& Mitchell, T. J. (1994). A role in the cell-binding for the C-terminus of pneumolysin, the thiol-activated toxin of Streptococcus pneumoniae. FEMS Microbiol Lett 121, 217-221.

Pinkney, M., Beachey, E. \& Kehoe, M. (1989). The thiol-activated toxin streptolysin $\mathrm{O}$ does not require a thiol group for cytolytic activity. Infect Immun 57, 2553-2558.

Rossjohn, J., Feil, S. C., McKinstry, W. J., Tweten, R. K. \& Parker, M. W. (1997). Structure of a cholesterol-binding, thiol-activated cytolysin and a model of its membrane form. Cell 89, 685-692.

Rossjohn, J., Gilbert, R. J. C., Crane, D. \& 7 other authors (1998). The molecular mechanism of pneumolysin, a virulence factor from Streptococcus pneumoniae. J Mol Biol 284, 449-461.

Saunders, F. K., Mitchell, T. J., Walker, J. A., Andrew, P. W. \& Boulnois, G. J. (1989). Pneumolysin, the thiol-activated toxin of Streptococcus pneumoniae, does not require a thiol group for in vitro activity. Infect Immun 57, 2547-2552.

Sekino-Suzuki, N., Nakamura, M., Mitsui, K.-I. \& Ohno-Iwashita, Y. (1996). Contribution of individual tryptophan residues to the structure and activity of $\theta$-toxin (perfringolysin $\mathrm{O}$ ), a cholesterolbinding cytolysin. Eur J Biochem 241, 941-947.

Shatursky, O., Heuck, A. P., Shepard, L. A., Rossjohn, J., Parker, M. W., Johnson, A. E. \& Tweten, R. K. (1999). The mechanism of membrane insertion for a cholesterol-dependent cytolysin: a novel paradigm for pore-forming toxins. Cell 99, 293-299.

Shepard, L. A., Heuck, A. P., Hamman, B. D., Rossjohn, J., Parker, M. W., Ryan, K. R., Johnson, A. E. \& Tweten, R. K. (1998). Identification of a membrane-spanning domain of the thiolactivated pore-forming toxin Clostridium perfringens perfringolysin $O$ : an $\alpha$-helical to $\beta$-sheet transition identified by fluorescence spectroscopy. Biochemistry 37, 14563-14574.

Shepard, L. A., Shatursky, O., Johnson, A. E. \& Tweten, R. K. (2000). The mechanism of pore assembly for a cholesteroldependent cytolysin: formation of a large prepore complex precedes the insertion of the transmembrane $\beta$-hairpins. Biochemistry 39, 10284-10293.

Shimada, Y., Nakamura, M., Naito, Y., Nomura, K. \& OhnoIwashita, Y. (1999). C-terminal amino acid residues are required for the folding and cholesterol binding property of perfringolysin O, a pore-forming cytolysin. J Biol Chem 274, 18536-18542.

Tweten, R. K., Parker, M. W. \& Johnson, A. E. (2001). The cholesterol-dependent cytolysins. Curr Top Microbiol Immunol 257, 15-33.

Received 7 June 2002; revised 9 August 2002; accepted 13 September 2002. 